\title{
Resection of Ewing Sarcoma in the Paranasal Sinus Via the Endoscopic Modified Lothrop (Draf III) Approach
}

\author{
Hye Min Han, MD, Young-Chan Kim, MD, Ki Jeong Lee, and Tae Hoon Kim, PhD \\ Department of Otorhinolaryngology-Head and Neck Surgery, Korea University, College of Medicine, Seoul, Korea
}

\begin{abstract}
Ewing sarcoma is a rare type of bone tumor that typically occurs in the head and neck regions. Appropriate management strategies remain controversial. Herein, we report a case of Ewing sarcoma originating from the ethmoid sinus in a 38-year-old woman. The patient initially presented with unilateral nasal obstruction. Pathologic examination revealed a tumor composed of small round blue cells under hematoxylin and eosin staining with positive expressions for CD-99, CK, and FLI-1. The patient was successfully treated with neoadjuvant chemotherapy followed by complete surgical excision via the endoscopic modified Lothrop (Draf III) approach, adjuvant chemotherapy, and radiation therapy. There was no recurrence or complication up to the writing of this article. This study presents an appropriate management strategy for an extensive case of Ewing sarcoma invading the frontal sinus using an endoscopic approach.
\end{abstract}

KEY WORDS: Ewing sarcoma $\cdot$ Paranasal sinus neoplasms · Ethmoid sinus · Frontal surgery.

\section{INTRODUCTION}

Ewing sarcoma is a rare type of malignant tumor, usually arising from the bone, and occurs in young patients. Ewing sarcoma of the head and neck region is uncommon and represents only $1-9 \%$ of all Ewing sarcoma cases. ${ }^{1)}$ As a result, appropriate management strategies remain controversial.

Herein, we report a case of localized Ewing sarcoma arising from the paranasal sinus that was successfully treated with the endoscopic modified Lothrop (Draf III) procedure with no recurrence or complication until the point of writing this article.

\section{CASE REPORT}

A 38-year-old woman was admitted to the Korea University Medical Center Oncology department. She presented with left nasal obstruction that developed approximately
1 month prior to initial assessment. Other accompanying symptoms included posterior nasal drip, hyposmia, and headache. The patient had no underlying disease apart from panic disorder and a surgical history of rhinoplasty 15 years ago.

The patient had visited another hospital immediately before admission and underwent left endoscopic sinus surgery. Biopsy from the left paranasal sinus showed poorly differentiated atypical round cells with necrosis that was consistent with a malignant tumor, such as malignant lymphoma and primitive neuroectodermal tumor. The Korea University Medical Center Pathology department reviewed the slide and reported small round cell tumor favoring Ewing sarcoma.

Initial endoscopic examination of the patient revealed a yellow polypoid mass arising from the left middle meatus. The left middle turbinate and nasal septum were deviated to the right due to the mass effect of the tumor (Fig. 1A).

Received: October 16, 2020 / Accepted: November 13, 2020

Address for correspondence: Tae Hoon Kim, MD, PhD, Department of Otorhinolaryngology-Head and Neck Surgery, Korea University, College of Medicine, Korea University Anam Hospital, 73 Goryedae-ro, Seongbuk-gu, Seoul 02841, Korea

Tel: +82-2-920-5486, Fax:+82-2-925-5233, E-mail: doctorthk@gmail.com 

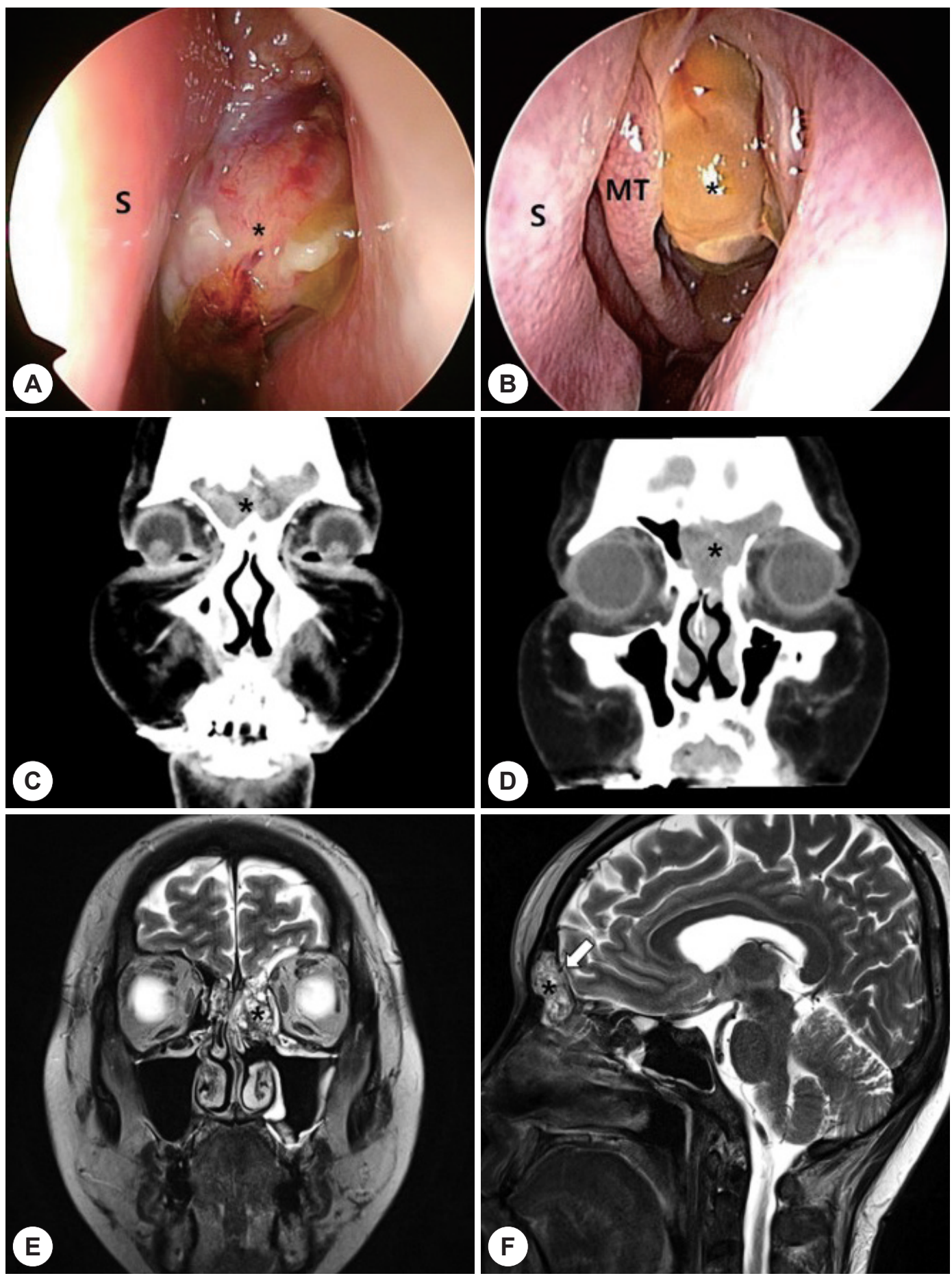

Fig. 1. Preoperative evaluation of the left ethmoidal sinus tumor. A: Endoscopic view of the left nasal cavity using a 0-degree rigid ENT endoscope during initial physical examination prior to any type of treatment. A huge polypoid mass originating from the left middle meatus (asterisk) can be seen. B: Endoscopic view of the left nasal cavity after 5 cycles of chemotherapy. A significant decrease in tumor size can be noted. The left middle turbinate (MT) and left nasal septum (S) are deviated to the right due to the tumor mass. C: Initial enhanced coronal computed tomography (CT) scan of the left paranasal sinus lesion (asterisk) involving the bilateral ethmoid and frontal sinuses. D: Enhanced coronal computed tomography (CT) scan of the left paranasal sinus lesion (asterisk) after 5 cycles of neoadjuvant chemotherapy demonstrates a significant decrease in tumor size. Additionally, a residual heterogeneously enhancing mass originating from the left ethmoid sinus region and involving the bilateral frontal and ethmoid sinuses can be identified. E: Enhanced magnetic resonance imaging (MRI) scan after 5 cycles of neoadjuvant chemotherapy demonstrates a heterogeneously enhancing T2 high signal intensity lesion (asterisk) as seen on the CT scan. F: Focal thinning of bilateral posterior bony wall of the frontal sinus (white arrow) on the sagittal plane image indicates possible focal bone erosion at the skull base. 
Additionally, a small septal perforation was noted. There was no palpable cervical neck node. Contrast-enhanced computed tomography (CT) of the neck area identified a heterogeneously enhancing mass measuring $47 \times 40 \times 25$ $\mathrm{mm}$, originating from the left ethmoid sinus and invading the bilateral frontal and ethmoidal sinuses. Magnetic resonance imaging (MRI) revealed bone erosion at the right frontal sinus posterior wall but no dural invasion (Fig. 1F). Chest CT and positron emission tomography (PET)-CT were negative for cervical lymphadenopathy and distant metastasis. The patient was diagnosed with localized Ewing's sarcoma of the paranasal sinus (cT3N0M0) and underwent 5 cycles of neoadjuvant chemotherapy comprising vincristine ( $2 \mathrm{mg} /$ day), adriamycin $\left(75 \mathrm{mg} / \mathrm{m}^{2} /\right.$ day) and cyclophosphamide $\left(1,200 \mathrm{mg} / \mathrm{m}^{2} /\right.$ day $)$ alternating with ifosfamide $\left(1,800 \mathrm{mg} / \mathrm{m}^{2} /\right.$ day $)$ and etoposide $\left(100 \mathrm{mg} / \mathrm{m}^{2} /\right.$ day $)$ (VAC/IE) over the course of 3 months.

Restaging after neoadjuvant chemotherapy treatment demonstrated a significant decrease in the tumor size. However, a small residual yellow cystic mass was observed in the left middle meatus (Fig. 1B). Paranasal CT revealed a slightly decreased extent of the tumor (Fig. 1D). The tumor measured $23 \times 34 \times 27 \mathrm{~mm}$. There were no significant changes in chest CT and PET-CT results (ycT2N0M0).

The patient underwent complete surgical removal of the remnant lesion via the endoscopic modified Lothrop (Draf III) approach (Fig. 2A). The extent of the tumor was examined endoscopically using the Scopis Hybrid Navigation System (Scopis Medical). The entire mass was removed using a debrider and ethmoid forceps. Invasion of the crista galli, anterior frontal sinus wall, and right posterior frontal sinus wall was noted. The bony origin of the tumor on the lateral wall of the left ethmoid sinus was drilled until the left periorbital fat was exposed. Bilateral middle turbinates and the upper septum were resected, and complete skull base skeletonization was performed. After complete removal of the tumor, skull base erosion was identified in the posterior wall of the right frontal sinus. The defect was reconstructed using nasal septal cartilage and mucosa from the right inferior turbinate. TachoComb and surgical glue was applied for further stability. Nasal packing was performed using both absorbable and nonabsorbable materials.

Pathological examination of the final specimen revealed a tumor comprising small round cells with a high nuclearto-cytoplasmic ratio, and the cells appeared blue on hematoxylin and eosin staining (Fig. 2B). The tumor showed membranous positivity for CD99, CK, and FLI-1 (Fig. 2C, D); focal positivity for chromogranin; and weak positivity for synaptophysin. The tumor was negative for LCA and Melan-A. However, dual-color fluorescence in situ hybrid-
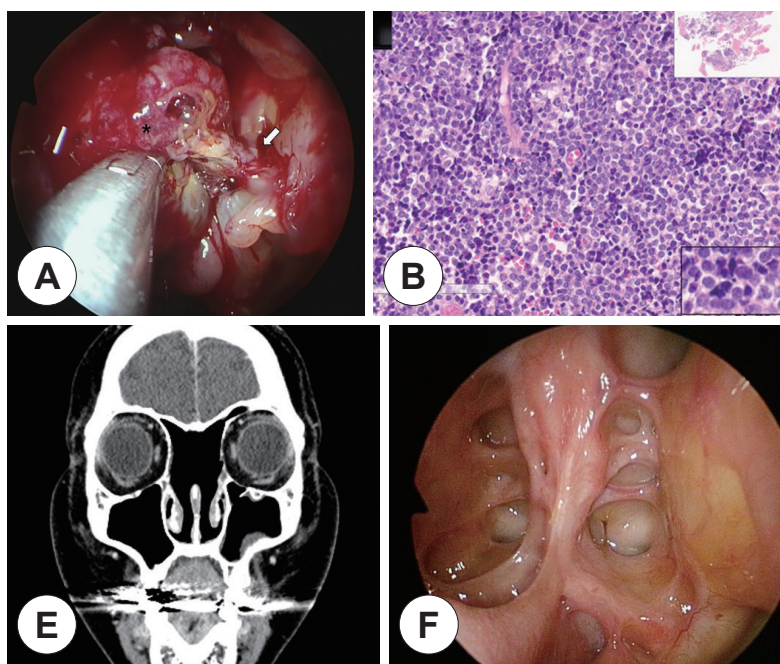
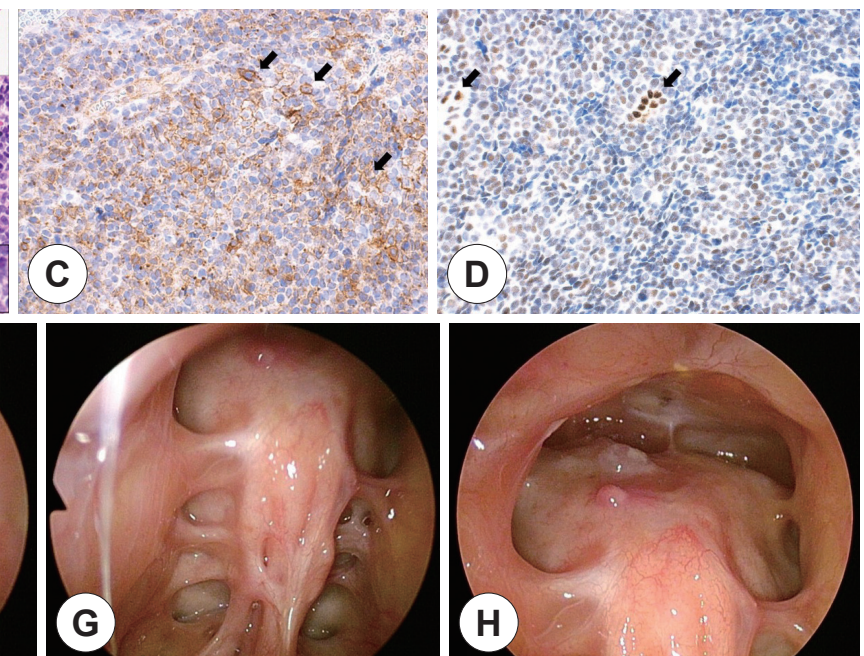

Fig. 2. Intraoperative view and postoperative pathologic, radiographic and endoscopic findings of left ethmoidal sinus tumor. A: Intraoperative view of the yellow polypoid mass (asterisk) originating from the left ethmoid sinus. The tumor seems to originate from the lateral wall of the left ethmoid sinus. The focal point of origin (white arrow) is marked with a Bovie coagulator for further drilling. B: Microscopic examination of the tumor under hematoxylin and eosin staining shows small round blue cells with a high nuclear-to-cytoplasmic ratio (H-E stain $\times 200$ ). C: CD-99 positive (black arrows). D: FLI-1 positive (black arrows). E: Coronal image of follow-up enhanced CT scan after 5 cycles of neoadjuvant chemotherapy, complete surgical resection, and adjuvant concurrent chemoradiation therapy ( 5 cycles of adjuvant chemotherapy and 5,500 cGy of radiation therapy). There is no visible evidence of residual or recurrent disease. F: Endoscopic view of the left post-ethmoidectomy OP bed from the left nasal cavity using a 70-degree rigid ENT endoscope. G: Endoscopic view of the right post-ethmoidectomy OP bed from the right nasal cavity using a 70-degree rigid ENT endoscope. H: Endoscopic view of the post-endoscopic frontal sinusotomy (Draf III) OP bed using a 70-degree rigid ENT endoscope. 
ization (FISH) performed with break apart rearrangement probe was negative for EWSR1 gene rearrangement $(10 \%$; cut-off value $11 \%$ ). The previously described tumor was found in the bilateral nasal cavity, intersinus septum, and left ethmoid sinus. Despite the negative FISH result, immunohistochemistry results indicated Ewing sarcoma in these regions.

The patient was stable without fever or cerebrospinal fluid (CSF) rhinorrhea. Postoperative care comprised prophylactic absolute bed rest for 1 week and Intravenous ceftriaxone ( 2 g IV2 for 14 days). Nasal packing was removed 3 days postoperatively. Adjuvant concurrent chemoradiation therapy was initiated 1 month after the surgery for another 5 cycles (a total of 10 cycles of VAC/IE), and adjuvant radiation therapy (total 5,500 cGy/31F) was administered to the nasal cavity for approximately 2 months.

The patient visited the otorhinolaryngology outpatient department for regular follow-ups after the surgery. A follow-up CT scan and physical examination including endoscopic examination of the nasal cavity displayed no evidence of tumor recurrence (Fig. 2E-H). At the most recent visit, which was 8 months after the endoscopic surgery when writing this article, the patient was stable without any noticeable discomfort or complication.

\section{DISCUSSION}

Ewing sarcoma is a malignancy that originates from the peripheral neuroectoderm and belongs to the Ewing sarcoma family of tumors that includes peripheral neuroectodermal tumors. Although Ewing sarcomas rarely occur in the skull or face, they tend to occur in the mandible, maxilla, skull, and sinonasal region. Vaccani et al. ${ }^{2)}$ reported that 5 in 70 cases $(7.1 \%)$ of Ewing's sarcoma involved the head and neck region.

The presenting symptoms typically comprise a slow growing, firm mass. Depending on the site of invasion, it may be associated with loose teeth, ${ }^{3)}$ otitis media, or exophthalmos. Lymphatic spread to the cervical region is uncommon. Advanced staging at the point of initial evaluation serves as the strongest negative prognostic factor. Initial evaluation should include CT and MRI of the primary site, chest $\mathrm{CT}$, and bone scan. Bone marrow biopsy or screening MRI of the spine and pelvis should be considered. Ewing sarcoma tumors are mostly lytic-sclerotic and show speculated periosteal reactions on plain films and CT scans. ${ }^{4}$
Histology and immunohistochemistry play key roles in the diagnosis of Ewing sarcoma. Ewing sarcoma is a type of small round cell tumor composed of sheets of small round blue cells owing to a high nuclear-to-cytoplasmic ratio. Ewing sarcoma shows positivity for CD99, FLI1, and neuronspecific enolase (NSE). ${ }^{5)}$ Moreover, identification of a reciprocal translocation involving EWSR1 (chromosome 22) and FLI1 (chromosome 11) may assist in the diagnosis of Ewing sarcoma. ${ }^{\text {) }}$

Here, we demonstrate a rare case of Ewing sarcoma that originated from the paranasal sinus. Although initially suspected as malignant lymphoma, subsequently, it was accurately diagnosed as Ewing sarcoma using appropriate immunohistochemistry studies. We would like to emphasize the possibility that these tumors may originate from the head and neck area, and hence, it is important to use appropriate techniques for accurate diagnosis and treatment.

Numerous studies have stated the importance of the tumor stage at the point of diagnosis as the most predictive prognostic variable. ${ }^{78)}$ and the long term survival rates were $<30 \%$ and $65-75 \%$ in patients with disseminated and localized disease, respectively. ${ }^{8)}$ As previous findings have suggested, the patient in this case was successfully treated for localized Ewing sarcoma with no recurrence 4 months post-treatment.

Treatment regimens for Ewing sarcomas of the head and neck remain controversial. Further studies on cases of successful local control of head and neck Ewing sarcomas are needed to establish a standardized treatment protocol. Currently, treatment regimens are mainly based on Ewing sarcomas that originate from regions such as the extremities and pelvis. The current literature suggests that treatment should be multimodal and include neoadjuvant or adjuvant chemotherapy and wide resection with reconstructive surgery, as needed. Moreover, combination radiotherapy may also be beneficial.

Chemotherapy can be administered as neoadjuvant or adjuvant therapy. Neoadjuvant chemotherapy aims to reduce the primary tumor burden and decrease the number and size of pulmonary metastasis. Adjuvant chemotherapy decreases postsurgical metastasis. ${ }^{9)}$ In this case, the patient initially presented with extensive disease, which seemed difficult to excise completely. Therefore, neoadjuvant chemotherapy was administered initially to decrease the primary tumor size to a surgically resectable size. Post-surgery, adjuvant chemotherapy was initiated to control postsurgi- 
cal recurrence and metastasis. Thus, the patient could gain the benefits of both methods.

The endoscopic modified Lothrop procedure consists of enlarging the frontonasal pathway by removing the frontal sinus floor, intersinus septum, superior nasal septum, and frontal beak. ${ }^{10)}$ To our knowledge, the majority of sinonasal Ewing sarcoma cases are treated with chemotherapy and radiation therapy owing to accessibility. Ewing sarcomas involving the paranasal sinuses require extensive external approaches. ${ }^{11-13)}$ Cases treated endoscopically typically involve tumors limited to the nasal cavity. ${ }^{14)}$ This case is significant as the Ewing sarcoma involving the frontal sinus was successfully resected using an endoscopic surgical method. We hope it provides a helpful example for other clinicians while making treatment decisions for difficult sinonasal Ewing sarcoma cases.

\section{Acknowledgments}

This research was supported by the Basic Science Research Program, National Research Foundation of Korea, funded by the Ministry of Science and Technology and the Ministry of Science, ICT \& Future Planning (2017R1A2B2003575, NRF-2020R1A2C1006398), and the Korea Health Technology R\&D Project (HI17C0387), Korea Health Industry Development Institute (KHIDI), and the Ministry of Health \& Welfare. This research was also supported by a Korea University grant, and a grant from Korea University Medical Center as well as by Anam Hospital, Seoul, Republic of Korea.

\section{REFERENCES}

1) Whaley JT, Indelicato DJ, Morris CG, Hinerman RW, Amdur RJ, Mendenhall WM, et al. Ewing tumors of the head and neck. Am J Clin Oncol 2010;33(4):321-6.
2) Vaccani JP, Forte V, de Jong AL, Taylor G. Ewing's sarcoma of the head and neck in children. International Journal of Pediatric Otorhinolaryngology 1999;48(3):209-16.

3) Sripathi Rao B, Rai G, Hassan S, Nadaf A. Ewing's sarcoma of the mandible. National Journal of Maxillofacial Surgery 2011;2(2):184-8.

4) Peersman B, Vanhoenacker FM, Heyman S, Herendael BV, Stam M, Brys P, et al. Ewing's sarcoma: imaging features. JBR-BTR 2007; 90(5):368-76.

5) Desai SS, Jambhekar NA. Pathology of Ewing's sarcoma/PNET: Current opinion and emerging concepts. Indian J Orthop 2010;44(4): 363-8.

6) Bailey K, Cost C, Davis I, Glade-Bender J, Grohar P, Houghton P, et al. Emerging novel agents for patients with advanced Ewing sarcoma: a report from the Children's Oncology Group (COG) New Agents for Ewing Sarcoma Task Force F1000Res 2019;8.

7) Burchill SA. Ewing's sarcoma: diagnostic, prognostic, and therapeutic implications of molecular abnormalities. J Clin Pathol 2003; 56(2):96-102.

8) Kovar H, Amatruda J, Brunet E, Burdach S, Cidre-Aranaz F, Enrique de Alava E, et al. The second European interdisciplinary Ewing sarcoma research summit--A joint effort to deconstructing the multiple layers of a complex disease. Oncotarget 2016;7(8):8613-24.

9) Ferguson JL, Turner SP. Bone Cancer: Diagnosis and Treatment Principles. American Family Physician 2018;98(4):205-13.

10) Shen J, Chan N, Wrobel BB. The endoscopic modified lothrop procedure: Review of single institution experience and long-term outcomes. Laryngoscope Investigative Otolaryngology 2018;3(2):105-9.

11) Agrawal A, Dulani R, Mahadevan A, Vagaha SJ, Vagha J, Shankar SK. Primary Ewing's sarcoma of the frontal bone with intracranial extension. Journal of Cancer Research and Therapeutics 2009;5(3): 208-9.

12) Alobid I, Bernal-Sprekelsen M, Alós L, Benítez P, Traserra J, Mullol J. Peripheral primitive neuroectodermal tumour of the left maxillary sinus. Acta Oto-Laryngologica 2003;123(6):776-8.

13) Thariat J, Italiano A, Peyrade F, Birtwisle-Peyrottes I, Gastaud L, Dassonville O, et al. Very Late Local Relapse of Ewing's Sarcoma of the Head and Neck treated with Aggressive Multimodal Therapy. Sarcoma 2008;2008:854141.

14) Lepera D, Volpi L, Facco C, Turri-Zanoni M, Battaglia P, Bernasconi B, et al. Endoscopic Treatment of Ewing Sarcoma of the Sinonasal Tract. The Journal of Craniofacial Surgery 2016;27(4):1001-6. 